\title{
Il processo della supervisione psicoanalitica. Apprendere la psicoanalisi o sperimentarla?
}

\author{
di Maria Luisa Tricoli \\ Giovanni Fioriti EdITORE, 2018
}

Non v'è procedura più difficile che insegnare la psicoanalisi senza il rischio di incorrere nel riduzionismo. E non v'è mestiere più impossibile del supervisore, senza incorrere nel rischio del paternalismo. La specificità' della tecnica del 'lavorare con le persone, per le persone, restando persone' obbliga a scegliere, costantemente, tra la tentazione di lasciarsi andare ad attitudini autoritarie e la capacità di accettare, da entrambe le parti (dello psicoanalista e del paziente/cliente) la sfida che raccontare e riflettere sulla interazione specialissima, insita nella pratica tra questi due esseri umani, comporta. Ed è forse proprio a causa di questa difficoltà (ma non solo) che la psicoanalisi, negli anni, ha ripetutamente tradito se' stessa, trasformandosi di conseguenza, nei contenuti e nei fatti, da evento trasgressivo e di 'rottura' delle istituzioni, sia terapeutiche che di pensiero, in una istituzione essa stessa, rigida ed aristocratica.

La 'prova' di siffatti tradimenti è in buona parte costituita dal suo essersi organizzata in Istituzione, ovvero il passaggio da Pensiero a Stato. La nascita degli Istituti, con la loro pluralità ne segna la trasformazione. Gli Istituti, per loro intrinseca natura sono fondati sull' 'appartenenza', e sulla 'affiliazione'. Appartenenza ad una 'forma' (la procedura psicoanalitica) e condivisione, non di rado acritica, dei contenuti così come formulati dai Padri Fondatori, Freud incluso. Vedasi p.es. le procedure di ammissione: cos'altro sono se non puri e semplici riti: numero minimo di sedute, appartenenza del didatta alla scuola, distinzione tra analisi personale ed analisi didattica (quasi fosse possibile)... E il rito, come noto, ad altro non serve che a sancire, fissare, stabilire... il Potere.

Avendo letto il libro, mi piace credere che la spinta a scriverlo sia stata per

*Scuola di Psicoterapia Conversazionale, Parma. E-mail: mariazirilli4747@gmail.com 
M. L. Tricoli, esattamente il contrario, ovvero il desiderio di 'mantenere' alla psicoanalisi il suo carattere di novità, alimentando vitalità e freschezza.

La 'rottura' ha inizio con il titolo (Il Processo della Supervisione Psicoanalitica) o, meglio, con i sottotitoli (Apprendere la psicoanalisi o sperimentarla?). Se il termine 'processo' allude ad una sequenza, rigida, di fatti ed in una qualche misura corrisponde ad 'apprendere' 'prendere qualcosa da qualcuno'; sperimentare contraddice la sequenza e la disperde. Un po' come se l'Autrice, accolta la propria disidentità, nel mentre che autorizza sé stessa ad 'asserire', contemporaneamente insinuasse la possibilità opposta del 'criticare'. Una attitudine al dubbio, tipica del ricercatore, che si ripropone, puntualmente all'inizio dei capitoli, tutti preceduti, contemporaneamente, da un titolo e da un sottotitolo che, inaspettatamente destabilizza. Tra tutti uno, poetico: Capitolo 4, dal titolo 'Vivere il processo candidato/paziente',

Sottotitolo:

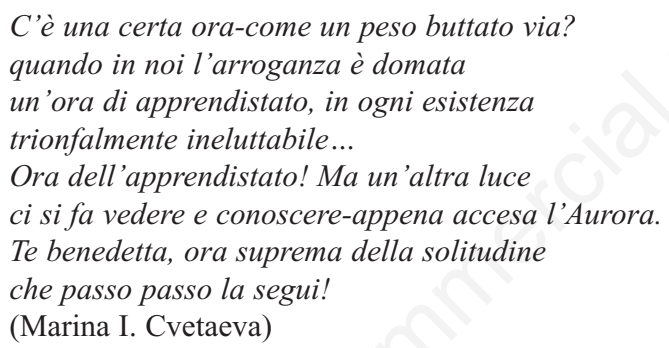

È nota, a chi insegna psicoanalisi, quanta e quale sia la paura che gli allievi maggiormente dotati sentono, e in parte trasmettono al loro mentore, allorché arrivano, dopo anni di studio teorico, sulle soglie del 'fare'. E 'fare da soli'. Un senso di angoscia che tocca solo ai migliori di loro, ai più sensibili, ai più dotati di empatia. Fare il supervisore implica un ossimoro. Se è vero che nel 'qui ed ora' della relazione medico-paziente ci sono soltanto due Soggetti, è altrettanto vero che la stanza di terapia, ospita una folla. Personaggi, storie, ricordi sia dell'uno che dell'altro, si mescolano e dialogano liberamente, soggetti a spinte sostanzialmente ignote, vera e propria Torre di Babele, con una sola 'via d'uscita': la ricerca, congiunta, del paziente e dell'analista, di un nuovo modo di raccontarsi (percepire, essere) e di raccontare (agire nel mondo). Fare il supervisore, essere 'terzo del terzo' implica, contemporaneamente, sia partecipazione (emotiva oltreché simbolica) che distanza.

È in questa sottolineatura della necessità' di mantenere viva l'emotività (la curiosità, l'interesse, la disponibilità' al cambiamento di sé stesso) intanto che si osserva (e si pensa), che risiede l'originalità del libro. Essere terzo implica un sapere 'entrare' e, contemporaneamente 'uscire' dallo specchio (Jaque Lacan). E per farlo, accanto al 'sapere' e al 'saper essere', si ha biso- 
gno di uno specchio. E non di uno specchio qualsiasi. Bensì dello 'specchio parlante', quello della strega di Biancaneve. Specchio quanto mai arduo e necessario. Per la sua obiettività, per il suo rigore, per la sua cattiveria, se necessaria.

Una posizione difficile che rende conto, tra l'altro del perché, come fortemente sostenuto da M. L. Tricoli, gli anziani, i supervisori, per non andare in frantumi per mano degli allievi e, specialmente dei pazienti, debbano mantenere costantemente il confronto e il dialogo col mondo delle idee, e naturalmente, con la societas, la cultura del momento, in tutte le sue articolazioni, ovvero sia con le altre branche della Medicina (prima fra tutte le Neuroscienze) e della Società (habitat, civitas).

M. L. Tricoli, nel suo libro lo fa costantemente, in controluce. Il libro, infatti, costruito come un manuale, (di cui si consiglia fortemente lo studio), in realtà è una sceneggiatura, una pièce autenticamente drammatica, nella quale M. L. Tricoli ha trasfuso conoscenza e dubbio, statuità e movimento.

Tant' è che sono costanti almeno due livelli di introiezione/lettura, sia sul piano strutturale (titolo e sottotitolo e/o premessa poetica ai vari capitoli) che sul piano più sostanziale, dei contenuti, esattamente corrispondenti alla storia classica del pensiero psicoanalitico ma anche puntualmente attraversati, tutti, dalla passione che connota l'autrice, per la ricerca di orizzonti, emotivi e simbolici, e saperi sempre nuovi.

Di tutto questo Tricoli è ben consapevole, tant'è che fin dai primi capitoli affronta il tema della trasmissione dei saperi all'interno degli Istituti:

'La formazione dovrebbe avere come compito basilare quello di suscitare il gusto per una tensione costante verso un ampliamento del sapere su di sé e sul proprio essere nel mondo'.

Dopo avere ripercorso, in chiave critica la storia del pensiero psicoanalitico, al riguardo approda senza remore al cuore del problema.

Oggi, nonostante gli sforzi di tanti (e tra i tanti di Kernberg cui si associa) la psicoanalisi non prevede una 'Teoria della tecnica' soddisfacente. 'La maggiore sfida attuale che riguarda non solo la supervisione, ma tutta la psicoanalisi odierna, è determinare quale 'comune teoria della tecnica' (common theory of technique) possa fornire agli studenti un solido terreno su cui тиоversi (Kernberg 2010), una sfida ancora aperta nonostante i tentativi di trovare un common ground (Wellenstein 1990) tra Psicologia dell'To, Kleiniani, Teoria della Relazione d'Oggetto e Relational track, che oggi va aggiunto ai tre precedenti poiché: 'la concezione costruttivista tipica della prospettiva relazionale, allorché ipotizza l'esistenza di una 'matrice relazionale' (costituita dalla relazione tra didatta e candidato) supera, de facto, l'ipotesi positivista della trasmissione di saperi precostituiti a favore di una co-costruzione del tutto nuova, ogni volta'(M. L. Tricoli, 2018), Da qui, la difficoltà di rappresentarla a sé stessi che di tradurla in una descrizione'. 
L'Autrice lo fa, con decisione e con umiltà. Con umiltà quando si adopera, sempre in prima battuta, a rappresentare le ipotesi che l'hanno preceduta (in un excursus minuzioso ed esaustivo) per introdurre subito dopo, e in chiusura, l'ipotesi relazionale (la sua) e le relative 'dimostrazioni' (casi clinici). Il valore del libro risiede appunto in questo, nell'aver saputo rappresentare un travaglio (quello proprio dell'Autrice) costantemente oscillante tra un 'sapere' consolidato e appreso, e la novità del paziente e del suo portavoce (l'allievo).

Personalmente, ho trovato magistrale la questione, per me più spinosa e difficile pagg. 117 e segg. paragrafo 2.3 relativa alla Diagnosi: non solo perché, la trattazione pur essendo dotta e completa risulta fluida ed accessibile, ma anche, e soprattutto, per il fatto che affronta con determinazione la questione 'se la psicoanalisi sia o meno scienza', e lo segnala in apertura, apponendo un sottotitolo al paragrafo: 'la capacità di comprendere prima di vedere, è il cuore del pensiero scientifico'.

Un po' come dire che la questione è mal posta, poiché 'fare psicoanalisi' è di per sé stesso una procedura scientifica che si dipana dalla intuizione alla Parola e al Simbolo.

Conflitto di interessi: l'autore dichiara che non vi sono potenziali conflitti di interessi.

Approvazione etica e consenso a partecipare: non necessario.

Ricevuto per la pubblicazione: 2 settembre 2021.

Accettato per la pubblicazione: 7 settembre 2021 .

${ }^{C}$ Copyright: the Author(s), 2021

Licensee PAGEPress, Italy

Ricerca Psicoanalitica 2021; XXXII:592

doi:10.4081/rp.2021.592

This article is distributed under the terms of the Creative Commons Attribution

Noncommercial License (by-nc 4.0) which permits any noncommercial use, distribution,

and reproduction in any medium, provided the original author(s) and source are credited. 